\title{
Integral cohomology of Hilbert schemes of points on surfaces
}

\author{
WeI-Ping Li AND Zhenbo QIN
}

Using Heisenberg algebra operators and interplay with the ring of symmetric functions, we verify a conjecture raised in [15]. As applications, we obtain linear bases and ring generators for the integral cohomology of the Hilbert schemes of points on a smooth projective surface with no odd cohomology.

\section{Introduction}

An additive basis for the rational cohomology group

$$
H^{*}\left(X^{[n]}\right)=\bigoplus_{i=0}^{4 n} H^{i}\left(X^{[n]}\right)
$$

of the Hilbert scheme $X^{[n]}$ of $n$ points on a projective surface $X$ can be given using the results of Göttsche, Grojnowski and Nakajima ([1-3]). When $X$ is a projective plane, Ellingsrud and Strømme [4] found a set of ring generators for the integral cohomology ring $H^{*}\left(X^{[n]} ; \mathbb{Z}\right)$, which essentially comes from the Chern classes of certain tautological bundles over $X^{[n]}$ (see [5] for a generalization). If $X$ is an arbitrary projective surface, a set of ring generators for the rational cohomology ring $H^{*}\left(X^{[n]}\right)$ is found in [6] (see also [7-11]). The result is for rational coefficients since the Chern characters of the tautological bundles were used in [6]. Recently, Markman [12-14] used the integral cohomology ring $H^{*}\left(X^{[n]} ; \mathbb{Z}\right)$ to study the weight-2 Hodge structure on $H^{2}(Y ; \mathbb{Z})$ when $X$ is a $K 3$ surface and $Y$ is a hyperkähler manifold deformation equivalent to $X^{[n]}$. It is interesting to search for additive bases and ring generators of the integral cohomology ring $H^{*}\left(X^{[n]} ; \mathbb{Z}\right)$ for a general surface $X$. In [15], an effective method for finding integral additive bases was developed. The main idea there is to study integral operators, i.e., linear operators on $H^{*}\left(X^{[n]}\right)$ which send integral classes in $H^{*}\left(X^{[n]}\right)$ to integral ones. 
Our goal in this paper is to determine both integral additive bases and integral ring generators when the odd Betti numbers of the surface $X$ vanish. To state our results, we introduce some notations first. Let

$$
\mathfrak{a}_{i}(\alpha), \quad i \in \mathbb{Z}, \alpha \in H^{*}(X)
$$

be the Heisenberg operators constructed in [16]. For a partition $\lambda$ of $n$ and a smooth curve $C$ in $X$, we can define the subvariety $L^{\lambda} C \subset X^{[n]}$ (see (3.1) and $[2,16])$. By Corollary 9.15 in $[16]$, the fundamental class $\left[L^{\lambda} C\right]$ can be written as $\mathfrak{m}_{\lambda, C}|0\rangle$ where $\mathfrak{m}_{\lambda, C}$ is a polynomial of creation operators $\mathfrak{a}_{-i}(C), i>0$. Therefore we can define the operator $\mathfrak{m}_{\lambda, \alpha}$ for an arbitrary class $\alpha \in H^{2}(X)$ simply by replacing the creation operators $\mathfrak{a}_{-i}(C), i>0$ appearing in $\mathfrak{m}_{\lambda, C}$ by the corresponding creation operators $\mathfrak{a}_{-i}(\alpha), i>0$. By Theorem 4.5 in [15], $\mathfrak{m}_{\lambda, \alpha}$ is integral when $\alpha$ is a divisor. It was conjectured in [15] that the linear operator $\mathfrak{m}_{\lambda, \alpha}$ is integral whenever $\alpha \in H^{2}(X)$ is an integral cohomology class.

In Section 3, we confirm the above conjecture, i.e., we prove that the operator $\mathfrak{m}_{\lambda, \alpha}$ is integral for every partition $\lambda$ and every integral class $\alpha \in$ $H^{2}(X)$. The main idea is to use topological $K$-theory and relations between the Hilbert schemes and the ring of symmetric functions. As an immediate consequence, we obtain the following (see the conventions below, (2.6) and (2.7) for notations).

Theorem 1.1. Let $X$ be a smooth projective surface with vanishing odd Betti numbers. Let $\alpha_{1}, \ldots, \alpha_{k}$ be an integral basis for $H^{2}(X ; \mathbb{Z}) /$ Tor. Then the classes

$$
\frac{1}{\mathfrak{z} \lambda} \mathfrak{a}_{-\lambda}(1) \mathfrak{a}_{-\mu}(x) \mathfrak{m}_{\nu^{1}, \alpha_{1}} \cdots \mathfrak{m}_{\nu^{k}, \alpha_{k}}|0\rangle, \quad|\lambda|+|\mu|+\sum_{i=1}^{k}\left|\nu^{i}\right|=n
$$

are integral, and furthermore, they form an integral basis for $H^{*}\left(X^{[n]} ; \mathbb{Z}\right) /$ Tor.

Theorem 1.1, which was also conjectured in [15], generalizes the corresponding statement in the Theorem 5.4 of [15] under the assumption that $H^{1}\left(X, \mathcal{O}_{X}\right)=H^{2}\left(X, \mathcal{O}_{X}\right)=0$.

In Section 4, we study the integral ring generators. Instead of using the Chern characters as in [6], we use the Chern classes of the tautological vector bundles $L^{[n]}$ over the Hilbert scheme $X^{[n]}$ where $L$ denotes line bundles over $X$ (see $(3.10)$ ). We investigate in details various relations among the Chern 
characters and Chern classes of $\mathcal{O}_{X}^{[n]}$, Heisenberg operators, and monomial symmetric functions. Using the techniques developed in $[15,17,18]$, we prove our second theorem.

Theorem 1.2. Suppose that the smooth projective surface $X$ has vanishing odd Betti numbers. Let $\alpha_{1}, \ldots, \alpha_{k}$ be an integral basis for $H^{2}(X ; \mathbb{Z}) /$ Tor. Then the integral ring $H^{*}\left(X^{[n]} ; \mathbb{Z}\right) /$ Tor is generated over $\mathbb{Z}$ by all the integral classes:

$$
c_{i}\left(\mathcal{O}_{X}^{[n]}\right), \quad \mathbf{1}_{-(n-j)} \mathfrak{m}_{\left(1^{j}\right), \alpha_{s}}|0\rangle, \quad \mathbf{1}_{-(n-j)} \mathfrak{a}_{-j}(x)|0\rangle
$$

where $1 \leq i \leq n-1,1 \leq j \leq n$, and $1 \leq s \leq k$.

The tautological rank- $n$ bundle $\mathcal{O}_{X}^{[n]}$ is defined by (3.10). The fundamental reason for the appearance of the classes $\mathbf{1}_{-(n-j)} \mathfrak{m}_{\left(1^{j}\right), \alpha_{s}}|0\rangle$ in the list (1.2) is the well-known fact (see [19]) that the monomial symmetric functions $m_{\left(1^{i}\right)}, i \geq 1$ corresponding to the partitions $\left(1^{i}\right), i \geq 1$ generate the (integral) ring of symmetric functions in infinitely many variables. We refer to Remark 4.2 for the geometric representations of the cohomology classes $\mathbf{1}_{-(n-j)} \mathfrak{m}_{\left(1^{j}\right), \alpha_{s}}|0\rangle$ and $\mathbf{1}_{-(n-j)} \mathfrak{a}_{-j}(x)|0\rangle$.

Finally, in view of the results in [4], we propose a conjecture concerning the ring generators of the integral ring $H^{*}\left(X^{[n]} ; \mathbb{Z}\right) /$ Tor in terms of certain Chern classes. Note that if $H^{1}\left(X, \mathcal{O}_{X}\right)=H^{2}\left(X, \mathcal{O}_{X}\right)=0$, then the odd Betti numbers of $X$ are equal to zero. Moreover, we can choose an integral basis $\alpha_{1}, \ldots, \alpha_{k}$ for $H^{2}(X ; \mathbb{Z}) /$ Tor such that $\alpha_{i} \cdot \alpha_{j}=1$ for some $i$ and $j$ with the possibility that $i=j$.

Conjecture 1.3. Assume that $H^{1}\left(X, \mathcal{O}_{X}\right)=H^{2}\left(X, \mathcal{O}_{X}\right)=0$. Let $\alpha_{1}, \ldots$, $\alpha_{k}$ be an integral basis for $H^{2}(X ; \mathbb{Z}) /$ Tor such that $\alpha_{i} \cdot \alpha_{j}=1$ for some $i$ and $j$. Put $\alpha_{k+1}=\alpha_{i}+\alpha_{j}$. Then the integral ring $H^{*}\left(X^{[n]} ; \mathbb{Z}\right) /$ Tor is generated over $\mathbb{Z}$ by:

$$
c_{i}\left(\mathcal{O}_{X}^{[n]}\right), \quad c_{j}\left(\mathcal{O}_{X}\left(\alpha_{s}\right)^{[n]}\right)
$$

where $1 \leq i \leq n-1,1 \leq j \leq n$, and $1 \leq s \leq(k+1)$.

Conventions. Unless otherwise indicated, all the cohomology groups in this paper are in $\mathbb{Q}$-coefficients. For a continuous map $p: Y_{1} \rightarrow Y_{2}$ between two smooth compact manifolds and for $\alpha_{1} \in H^{*}\left(Y_{1}\right)$, we define $p_{*}\left(\alpha_{1}\right)$ to be $\mathrm{PD}^{-1} p_{*}\left(\mathrm{PD}\left(\alpha_{1}\right)\right)$ where $\mathrm{PD}$ stands for the Poincaré duality. For a smooth 
projective surface $X$, by abusing notations, we let $1 \in H^{0}(X ; \mathbb{Z})$ be the fundamental cohomology class of $X$; in addition, we let $x$ denote either a point in $X$, or the class in $H^{4}(X ; \mathbb{Z})$ which is the Poincaré dual of the homology class represented by a point in $X$.

\section{Basics on Hilbert schemes of points on surfaces}

Let $X$ be a complex smooth projective surface, and $X^{[n]}$ be the Hilbert scheme of points on $X$. An element in $X^{[n]}$ is represented by a length- $n$ 0-dimensional closed subscheme $\xi$ of $X$. It is well known that $X^{[n]}$ is smooth. Sending an element in $X^{[n]}$ to its support in the symmetric product $\operatorname{Sym}^{n}(X)$, we obtain the Hilbert-Chow morphism $\pi_{n}: X^{[n]} \rightarrow \operatorname{Sym}^{n}(X)$, which is a resolution of singularities. Let $H^{*}\left(X^{[n]}\right)$ be the total cohomology of $X^{[n]}$ with $\mathbb{Q}$-coefficients, and put

$$
\mathbb{H}_{X}=\bigoplus_{n=0}^{\infty} H^{*}\left(X^{[n]}\right)
$$

There exists a non-degenerate super-symmetric bilinear form $\langle-,-\rangle$ on the space $\mathbb{H}_{X}$ which is induced from the standard one on $H^{*}\left(X^{[n]}\right)$ defined by

$$
\langle\alpha, \beta\rangle=\int_{X^{[n]}} \alpha \beta, \quad \alpha, \beta \in H^{*}\left(X^{[n]}\right) .
$$

Recall that Heisenberg algebra action on $\mathbb{H}_{X}$ was defined in $[2,3,16]$. Namely, we have the operators $\mathfrak{a}_{-n}(\alpha) \in \operatorname{End}\left(\mathbb{H}_{X}\right)$ with $n \in \mathbb{Z}$ and $\alpha \in$ $H^{*}(X)$ which satisfy a Heisenberg algebra commutation relation:

$$
\left[\mathfrak{a}_{m}(\alpha), \mathfrak{a}_{n}(\beta)\right]=-m \delta_{m,-n} \cdot\langle\alpha, \beta\rangle \cdot \operatorname{Id}_{\mathbb{H}_{X}} \cdot
$$

When $n>0$, we often refer to $\mathfrak{a}_{-n}(\alpha)$ (resp. $\mathfrak{a}_{n}(\alpha)$ ) as the creation (resp. annihilation) operator. The space $\mathbb{H}_{X}$ is an irreducible module over the Heisenberg algebra generated by the operators $\mathfrak{a}_{n}(\alpha)$ with a highest weight vector

$$
|0\rangle=1 \in H^{0}\left(X^{[0]}\right) \cong \mathbb{Q}
$$

It follows that $\mathbb{H}_{X}$ is linearly spanned by all the Heisenberg monomial classes:

$$
\mathfrak{a}_{-n_{1}}\left(\alpha_{1}\right) \cdots \mathfrak{a}_{-n_{k}}\left(\alpha_{k}\right)|0\rangle
$$

where $k \geq 0, n_{1}, \ldots, n_{k}>0$, and $\alpha_{1}, \ldots, \alpha_{k}$ run over a linear basis of $H^{*}(X)$. 
For $n>0$ and a homogeneous class $\alpha \in H^{*}(X)$, let $|\alpha|=s$ if $\alpha \in H^{s}(X)$, and let $G_{i}(\alpha, n)$ be the homogeneous component in $H^{|\alpha|+2 i}\left(X^{[n]}\right)$ of

$$
G(\alpha, n)=p_{1 *}\left(\operatorname{ch}\left(\mathcal{O}_{\mathcal{Z}_{n}}\right) \cdot p_{2}^{*} \operatorname{td}(X) \cdot p_{2}^{*} \alpha\right) \in H^{*}\left(X^{[n]}\right)
$$

where $\mathcal{Z}_{n}$ is the universal codimension-2 subscheme defined by

$$
\mathcal{Z}_{n}=\left\{(\xi, x) \subset X^{[n]} \times X \mid x \in \operatorname{Supp}(\xi)\right\} \subset X^{[n]} \times X
$$

$\operatorname{ch}\left(\mathcal{O}_{\mathcal{Z}_{n}}\right)$ denotes the Chern character of the structure sheaf $\mathcal{O}_{\mathcal{Z}_{n}}, \operatorname{td}(X)$ denotes the Todd class, and $p_{1}$ and $p_{2}$ are the projections of $X^{[n]} \times X$ to $X^{[n]}$ and $X$, respectively. We extend the notion $G_{i}(\alpha, n)$ linearly to an arbitrary class $\alpha \in H^{*}(X)$, and set $G_{i}(\alpha, 0)=0$. These Chern character classes $G_{i}(\alpha, n)$ play essential roles in understanding the cup product on $X^{[n]}$.

Let $\lambda=\left(1^{m_{1}} 2^{m_{2}} \ldots\right)$ be a partition of the integer $n=\sum_{r} r m_{r}$, whose part $r$ has multiplicity $m_{r}$. We write $\lambda \vdash n$. Let $\ell(\lambda)=\sum_{r} m_{r}$ and

$$
\begin{aligned}
|\lambda| & =\sum_{r} r m_{r}=n, \\
\mathfrak{z} \lambda & =\prod_{r \geq 1}\left(r^{m_{r}} m_{r} !\right) .
\end{aligned}
$$

In addition, for a cohomology class $\alpha \in H^{*}(X)$ and $m \geq 0$, we define

$$
\begin{aligned}
\mathfrak{a}_{-\lambda}(\alpha) & =\prod_{r \geq 1} \mathfrak{a}_{-r}(\alpha)^{m_{r}} \\
\mathbf{1}_{-m} & =\frac{1}{m !} \cdot \mathfrak{a}_{-\left(1^{m}\right)}(1)=\frac{1}{m !} \cdot \mathfrak{a}_{-1}(1)^{m} .
\end{aligned}
$$

The geometric meaning of $\mathbf{1}_{-m}$ is that $\mathbf{1}_{-m}|0\rangle$ is equal to the fundamental class of the Hilbert scheme $X^{[m]}$. For simplicity, we set $\mathbf{1}_{-m}=0$ when $m<0$.

The following is the Stability Theorem 5.1 proved in [18], which will be a key technical tool used in later sections.

Theorem 2.1. Let $s \geq 1$ and $k_{i} \geq 1$ for $1 \leq i \leq s$. Fix $n_{i, j} \geq 1$ and $\alpha_{i, j} \in$ $H^{*}(X)$ for $1 \leq j \leq k_{i}$, and fix $n$ with $n \geq \sum_{j=1}^{k_{i}} n_{i, j}$ for all $1 \leq i \leq s$. Then 
the cup product

$$
\prod_{i=1}^{s}\left(\mathbf{1}_{-\left(n-\sum_{j=1}^{k_{i}} n_{i, j}\right)}\left(\prod_{j=1}^{k_{i}} \mathfrak{a}_{-n_{i, j}}\left(\alpha_{i, j}\right)\right)|0\rangle\right)
$$

in $H^{*}\left(X^{[n]}\right)$ is equal to a finite linear combination of monomials of the form

$$
\mathbf{1}_{-\left(n-\sum_{p=1}^{N} m_{p}\right)}\left(\prod_{p=1}^{N} \mathfrak{a}_{-m_{p}}\left(\gamma_{p}\right)\right)|0\rangle
$$

whose coefficients are independent of $X, \alpha_{i, j}$ and $n$. Here $\sum_{p=1}^{N} m_{p} \leq$ $\sum_{i=1}^{s} \sum_{j=1}^{k_{i}} n_{i, j}$, and $\gamma_{1}, \ldots, \gamma_{N}$ depend only on $\alpha_{i, j}, 1 \leq i \leq s, 1 \leq j \leq k_{i}$, and the canonical class and the Euler class of $X$. In addition, the expression (2.9) satisfies the upper bound

$$
\sum_{p=1}^{N} m_{p}=\sum_{i=1}^{s} \sum_{j=1}^{k_{i}} n_{i, j}
$$

if and only if it is $\mathbf{1}_{-\left(n-\sum_{i=1}^{s} \sum_{j=1}^{k_{i}} n_{i, j}\right)}\left(\prod_{i=1}^{s} \prod_{j=1}^{k_{i}} \mathfrak{a}_{-n_{i, j}}\left(\alpha_{i, j}\right)\right)|0\rangle$ with coefficient 1 .

In particular, Theorem 2.1 says that the cup product (2.8) is of the form:

$$
\mathbf{1}_{-\left(n-\sum_{i=1}^{s} \sum_{j=1}^{k_{i}} n_{i, j}\right)}\left(\prod_{i=1}^{s} \prod_{j=1}^{k_{i}} \mathfrak{a}_{-n_{i, j}}\left(\alpha_{i, j}\right)\right)|0\rangle+\mathbf{1}_{-\left(n-\sum_{i=1}^{s} \sum_{j=1}^{k_{i}} n_{i, j}+1\right)} A
$$

for some cohomology class $A$ in $H^{*}\left(X^{\left[\sum_{i=1}^{s} \sum_{j=1}^{k_{i}} n_{i, j}-1\right]}\right)$.

\section{Integral basis of the integral cohomology}

In this section, using the method developed in [15] and topological $K$-theory, we will prove a conjecture raised in [15]. As a consequence, we will find an integral basis for $H^{*}\left(X^{[n]} ; \mathbb{Z}\right) /$ Tor, and hence prove Theorem 1.1.

\subsection{Integral operators and the definition of $\mathfrak{m}_{\lambda, \alpha}$}

In this subsection, we review the basic results and methods from [15]. 


\section{Definition 3.1.}

(i) A class $A \in H^{*}\left(X^{[n]}\right)$ is integral if it is contained in

$$
H^{*}\left(X^{[n]} ; \mathbb{Z}\right) / \text { Tor } \subset H^{*}\left(X^{[n]}\right) .
$$

(ii) A linear basis of $H^{*}\left(X^{[n]}\right)$ is integral if its members are integral classes and form a $\mathbb{Z}$-basis of the lattice $H^{*}\left(X^{[n]} ; \mathbb{Z}\right) /$ Tor.

(iii) A linear operator $\mathfrak{f} \in \operatorname{End}\left(\mathbb{H}_{X}\right)$ is integral if $\mathfrak{f}(A) \in \mathbb{H}_{X}$ is an integral class whenever $A \in \mathbb{H}_{X}$ is an integral cohomology class.

The following lemma compiles various results proved in [15].

\section{Lemma 3.2.}

(i) The operators $\mathfrak{a}_{n}(\alpha), n \in \mathbb{Z}$ are integral if $\alpha \in H^{*}(X)$ is integral;

(ii) The operator $1 / \mathfrak{z}_{\lambda} \cdot \mathfrak{a}_{-\lambda}(1)$ is integral for every partition $\lambda$;

(iii) Let $A \in \mathbb{H}_{X}$ be an integral class. Write $A$ as $\mathfrak{a}_{A}|0\rangle$ where $\mathfrak{a}_{A}$ is a unique polynomial of creation operators. Then, $\mathfrak{a}_{A}$ is an integral operator.

Next, let $C$ be a smooth irreducible curve in the surface $X$. By abusing notations, we also use $C$ to denote the corresponding divisor and the corresponding cohomology class. Following Subsection 9.3 of [16] (see also [2]), for every partition $\lambda=\left(\lambda_{1}, \lambda_{2}, \ldots\right)$ of $n$, we define the subvariety of $X^{[n]}$ :

$$
L^{\lambda} C=\overline{\left(\pi_{n}\right)^{-1}\left(S_{\lambda}^{n} C\right)}
$$

where $S_{\lambda}^{n} C=\left\{\sum_{i} \lambda_{i} x_{i} \mid x_{i} \in C, x_{i} \neq x_{j}\right.$ for $\left.i \neq j\right\}$, and

$$
\pi_{n}: X^{[n]} \rightarrow \operatorname{Sym}^{n}(X)
$$

is the Hilbert-Chow morphism. For $n \geq 0$, let $\mathbb{H}_{n, C}$ be the $\mathbb{Q}$-linear span:

$$
\operatorname{Span}\left\{\mathfrak{a}_{-\lambda}(C)|0\rangle \mid \lambda \vdash n\right\}
$$

in $H^{*}\left(X^{[n]}\right)$. We see from the Theorem 9.14 in [16] that the fundamental class $\left[L^{\lambda} C\right] \in H^{2 n}\left(X^{[n]} ; \mathbb{Z}\right)$ is contained in $\mathbb{H}_{n, C} \subset H^{*}\left(X^{[n]}\right)$. Define

$$
\mathbb{H}_{C}=\bigoplus_{n=0}^{\infty} \mathbb{H}_{n, C}
$$


Let $\Lambda$ be the ring of symmetric functions in infinitely many variables (see $\left[19\right.$, p.19]), and $\Lambda_{\mathbb{Q}}=\Lambda \otimes_{\mathbb{Z}} \mathbb{Q}$. For a partition $\lambda$, let $p_{\lambda}$ and $m_{\lambda}$ be the power-sum symmetric function and the monomial symmetric function, respectively.

In [16], Nakajima defined a linear isomorphism

$$
\Phi_{C}: \Lambda_{\mathbb{Q}} \longrightarrow \mathbb{H}_{C},
$$

which satisfies the following two properties:

$$
\Phi_{C}\left(p_{\lambda}\right)=\mathfrak{a}_{-\lambda}(C)|0\rangle, \quad \Phi_{C}\left(m_{\lambda}\right)=\left[L^{\lambda} C\right] .
$$

By Lemma 3.2 (iii), the operator $\mathfrak{a}_{\left[L^{\lambda} C\right]}$ is integral. Put

$$
\mathfrak{m}_{\lambda, C}=\mathfrak{a}_{\left[L^{\lambda} C\right]} \in \operatorname{End}\left(\mathbb{H}_{X}\right) .
$$

Since $\left[L^{\lambda} C\right] \in \mathbb{H}_{|\lambda|, C}$, we see from (3.2) that the integral operator $\mathfrak{m}_{\lambda, C}$ is a polynomial of the creation Heisenberg operators $\mathfrak{a}_{-i}(C), i>0$.

Definition 3.3. For a partition $\lambda$ and an arbitrary class $\alpha \in H^{2}(X)$, we define

$$
\mathfrak{m}_{\lambda, \alpha} \in \operatorname{End}\left(\mathbb{H}_{X}\right)
$$

by replacing the creation operators $\mathfrak{a}_{-i}(C)$ in $\mathfrak{m}_{\lambda, C}$ by the corresponding creation operators $\mathfrak{a}_{-i}(\alpha)$. Define the cohomology class $\left[L^{\lambda} \alpha\right] \in H^{*}\left(X^{[|\lambda|]}\right)$ by putting

$$
\left[L^{\lambda} \alpha\right]=\mathfrak{m}_{\lambda, \alpha}|0\rangle .
$$

Similarly, we can define the subspaces $\mathbb{H}_{n, \alpha}$ and $\mathbb{H}_{\alpha}$ of $\mathbb{H}_{X}$ as in (3.3). Moreover, in view of (3.4) and (3.5), we have a linear isomorphism

$$
\Phi_{\alpha}: \Lambda_{\mathbb{Q}} \longrightarrow \mathbb{H}_{\alpha}
$$

which satisfies the following two properties:

$$
\Phi_{\alpha}\left(p_{\lambda}\right)=\mathfrak{a}_{-\lambda}(\alpha)|0\rangle, \quad \Phi_{\alpha}\left(m_{\lambda}\right)=\left[L^{\lambda} \alpha\right]=\mathfrak{m}_{\lambda, \alpha}|0\rangle .
$$

Since the monomial symmetric function $m_{\left(1^{n}\right)}$ is the same as the $n$th elementary symmetric function, we see from formula $\left(2.14^{\prime}\right)$ in [19] that

$$
\mathfrak{m}_{\left(1^{n}\right)}=\sum_{|\mu|=n} \frac{(-1)^{n-\ell(\mu)} p_{\mu}}{\mathfrak{z}_{\mu}} .
$$


Therefore, applying $\Phi_{\alpha}$ to both sides and using (3.8), we obtain

$$
\mathfrak{m}_{\left(1^{n}\right), \alpha}|0\rangle=\left[L^{\left(1^{n}\right)} \alpha\right]=\sum_{|\mu|=n} \frac{(-1)^{n-\ell(\mu)} \mathfrak{a}_{-\mu}(\alpha)|0\rangle}{\mathfrak{z}_{\mu}} .
$$

\subsection{Integrality of $\mathfrak{m}_{\lambda, \alpha}$ for integral $\alpha$}

In this subsection, we shall prove that if $\alpha \in H^{2}(X)$ is integral, then the class $\left[L^{\lambda} \alpha\right]$ and the operator $\mathfrak{m}_{\lambda, \alpha}$ are integral. This was proved in Theorem 4.5 of [15] when $\alpha$ is a divisor, and conjectured in Remark 5.6 of [15] for a general integral class $\alpha$.

First of all, we recall some tautological vector bundles over the Hilbert scheme $X^{[n]}$. For a line bundle $L$ over the surface $X$, we define

$$
L^{[n]}=\left(p_{1}\right)_{*}\left(\mathcal{O}_{\mathcal{Z}_{n}} \otimes p_{2}^{*} L\right)
$$

where $\mathcal{Z}_{n}$ is from $(2.5)$, and $p_{1}$ and $p_{2}$ are the projections of $X^{[n]} \times X$ to $X^{[n]}$ and $X$, respectively. Then, $L^{[n]}$ is a rank- $n$ bundle over $X^{[n]}$.

Lemma 3.4. Let $0 \leq i \leq n$. Let $L$ be a line bundle on $X$ with $\alpha=c_{1}(L)$. Then,

$$
c_{i}\left(L^{[n]}\right)=\sum_{\substack{|\lambda|+|\mu|=n \\ \ell(\lambda)=n-i}} \frac{(-1)^{i-\ell(\mu)} \mathfrak{a}_{-\lambda}(1) \mathfrak{a}_{-\mu}(\alpha)|0\rangle}{\mathfrak{z} \lambda \mathfrak{z}_{\mu}}
$$

Proof. Let $c_{\hbar}\left(L^{[n]}\right)=\sum_{i=0}^{n} c_{i}\left(L^{[n]}\right) \hbar^{i}$ be the generating function for the Chern classes of $L^{[n]}$, where $\hbar$ is a formal variable. Then the Theorem 2.4 of [20], which is a variant of the Theorem 4.6 in [7], states that

$$
\sum_{n=0}^{\infty} c_{\hbar}\left(L^{[n]}\right) w^{n}=\exp \left(\sum_{r \geq 1} \frac{(-\hbar)^{r-1}}{r} \mathfrak{a}_{-r}\left(c_{\hbar}(L)\right) w^{r}\right) \cdot|0\rangle
$$


where $w$ is another formal variable. Expanding the right-hand side yields

$$
\begin{aligned}
& \exp \left(\sum_{r \geq 1} \frac{(-\hbar)^{r-1}}{r} \mathfrak{a}_{-r}\left(c_{\hbar}(L)\right) w^{r}\right) \cdot|0\rangle \\
& =\exp \left(\sum_{r \geq 1} \frac{(-1)^{r-1}}{r} \mathfrak{a}_{-r}(1) \hbar^{r-1} w^{r}\right) \cdot \exp \left(\sum_{r \geq 1} \frac{(-1)^{r-1}}{r} \mathfrak{a}_{-r}(\alpha) \hbar^{r} w^{r}\right) \cdot|0\rangle \\
& =\sum_{\lambda} \frac{(-1)^{|\lambda|-\ell(\lambda)} \mathfrak{a}_{-\lambda}(1)}{\mathfrak{z} \lambda} \hbar^{|\lambda|-\ell(\lambda)} w^{|\lambda|} \cdot \sum_{\mu} \frac{(-1)^{|\mu|-\ell(\mu)} \mathfrak{a}_{-\mu}(\alpha)}{\mathfrak{z}_{\mu}} \hbar^{|\mu|} w^{|\mu|}|0\rangle \\
& =\sum_{(\lambda, \mu)} \frac{(-1)^{|\lambda|+|\mu|-\ell(\lambda)-\ell(\mu)} \mathfrak{a}_{-\lambda}(1) \mathfrak{a}_{-\mu}(\alpha)|0\rangle}{\mathfrak{z} \lambda \mathfrak{z}_{\mu}} \hbar^{|\lambda|+|\mu|-\ell(\lambda)} w^{|\lambda|+|\mu|}
\end{aligned}
$$

Now the Chern class $c_{i}\left(L^{[n]}\right)$ is the coefficients of $\hbar^{i} w^{n}$ in (3.13).

Lemma 3.5. If the class $\alpha \in H^{2}(X)$ is integral, then so is the operator $\mathfrak{m}_{\left(1^{n}\right), \alpha}$.

Proof. Let $p_{1}$ and $p_{2}$ be the projections of $X^{[n]} \times X$ to $X^{[n]}$ and $X$, respectively. By Theorem in [21, p. 20], $p_{1}$ induces a homomorphism

$$
\left(p_{1}\right) !: K_{\text {top }}^{0}\left(X^{[n]} \times X\right) \rightarrow K_{\text {top }}^{0}\left(X^{[n]}\right),
$$

where $K_{\text {top }}^{0}(\cdot)$ denotes the ring of complex vector bundles; moreover, for an element $V \in K_{\text {top }}^{0}\left(X^{[n]} \times X\right)$, the Riemann-Roch formula holds:

$$
\operatorname{ch}\left(\left(p_{1}\right) !(V)\right)=\left(p_{1}\right)_{*}\left(\operatorname{ch}(V) \cdot p_{2}^{*} \operatorname{td}(X)\right)
$$

Next, by Theorem E.5 in [22], there exists a complex (not necessarily algebraic) line bundle $L_{\alpha}$ on $X$ with $c_{1}\left(L_{\alpha}\right)=\alpha$. Applying (3.14) to the element

$$
V=V_{\alpha}:=\mathcal{O}_{\mathcal{Z}_{n}} \otimes p_{2}^{*} L_{\alpha} \in K_{\text {top }}^{0}\left(X^{[n]} \times X\right)
$$

where $\mathcal{Z}_{n}$ is the universal codimension-2 subscheme defined in (2.5), we obtain

$$
\begin{aligned}
\operatorname{ch}\left(\left(p_{1}\right)_{!}\left(V_{\alpha}\right)\right) & =p_{1 *}\left(\operatorname{ch}\left(\mathcal{O}_{\mathcal{Z}_{n}}\right) \cdot p_{2}^{*} \operatorname{td}(X) \cdot p_{2}^{*} \operatorname{ch}\left(L_{\alpha}\right)\right) \\
& =G(1, n)+G(\alpha, n)+\langle\alpha, \alpha\rangle / 2 \cdot G(x, n),
\end{aligned}
$$


where we have used definition (2.4) and the fact that

$$
\operatorname{ch}\left(L_{\alpha}\right)=1+\alpha+\langle\alpha, \alpha\rangle / 2 \cdot x .
$$

In particular, we conclude that the integral class $c_{i}\left(\left(p_{1}\right) !\left(V_{\alpha}\right)\right)$ is a polynomial $P_{i}$ of $G_{i_{1}}(1, n), G_{i_{2}}(\alpha, n),\langle\alpha, \alpha\rangle$ and $G_{i_{3}}(x, n)$ with $0 \leq i_{1}, i_{2}, i_{3} \leq 2 n$, i.e.,

$c_{i}\left(\left(p_{1}\right)_{!}\left(V_{\alpha}\right)\right)=P_{i}\left(G_{i_{1}}(1, n), G_{i_{2}}(\alpha, n),\langle\alpha, \alpha\rangle, G_{i_{3}}(x, n) \mid 0 \leq i_{1}, i_{2}, i_{3} \leq 2 n\right)$.

Note that the coefficients in $P_{i}$ are independent of $\alpha$ and $X$.

When $\alpha$ is a divisor, $L_{\alpha}$ is an algebraic line bundle on $X$, and the element

$$
\left(p_{1}\right) !\left(V_{\alpha}\right) \in K_{\text {top }}^{0}\left(X^{[n]}\right)
$$

is represented precisely by the tautological rank- $n$ vector bundle $\left(L_{\alpha}\right)^{[n]}$ defined in (3.10). Therefore, we obtain from Lemma 3.4 that

$$
c_{i}\left(\left(p_{1}\right) !\left(V_{\alpha}\right)\right)=\sum_{\substack{|\lambda|+|\mu|=n \\ \ell(\lambda)=n-i}} \frac{(-1)^{i-\ell(\mu)} \mathfrak{a}_{-\lambda}(1) \mathfrak{a}_{-\mu}(\alpha)|0\rangle}{\mathfrak{z} \lambda \mathfrak{z} \mu}
$$

Finally, we conclude from (3.16) and the universality results in [18] (e.g., the Theorem 4.1 there) that (3.17) holds for a general integral class $\alpha \in$ $H^{2}(X)$. Setting $i=n$ in (3.17) and using (3.9), we obtain the integral class

$$
c_{n}\left(\left(p_{1}\right) !\left(V_{\alpha}\right)\right)=\sum_{|\mu|=n} \frac{(-1)^{n-\ell(\mu)} \mathfrak{a}_{-\mu}(\alpha)|0\rangle}{\mathfrak{z}_{\mu}}=\left[L^{\left(1^{n}\right)} \alpha\right] .
$$

By Lemma 3.2 (iii) and (3.6), the operator $\mathfrak{m}_{\left(1^{n}\right), \alpha}$ is integral.

Theorem 3.6. Let the class $\alpha \in H^{2}(X)$ be integral. Then for every partition $\lambda$, the class $\left[L^{\lambda} \alpha\right]$ and the operator $\mathfrak{m}_{\lambda, \alpha}$ are integral.

Proof. It is well known (see [19]) that the monomial symmetric functions

$$
m_{\left(1^{i}\right)}, \quad i \geq 1
$$

generate the integral ring $\Lambda$. So the monomial symmetric function $m_{\lambda}$ can be written as an integral polynomial $P$ of the functions $m_{\left(1^{i}\right)}, i \geq 1$, i.e.,

$$
m_{\lambda}=P\left(m_{\left(1^{i}\right)} \mid i \geq 1\right) .
$$


Combining this with the two formulas in (3.8), we see that

$$
\left[L^{\lambda} \alpha\right]=\Phi_{\alpha}\left(m_{\lambda}\right)=\Phi_{\alpha}\left(P\left(m_{\left(1^{i}\right)} \mid i \geq 1\right)\right)=P\left(\mathfrak{m}_{\left(1^{i}\right), \alpha} \mid i \geq 1\right)|0\rangle .
$$

Hence, the class $\left[L^{\lambda} \alpha\right]$ is integral by Lemma 3.5. Finally, we conclude from Lemma 3.2 (iii) and (3.6) that the operator $\mathfrak{m}_{\lambda, \alpha}$ is integral.

\subsection{Proof of Theorem 1.1}

Let $\alpha_{1}, \ldots, \alpha_{k} \in H^{2}(X)$ be integral cohomology classes. By Lemma 3.2 and Theorem 3.6, we have three types of integral operators:

$$
\frac{1}{\mathfrak{z} \lambda} \mathfrak{a}_{-\lambda}(1), \quad \mathfrak{a}_{-\mu}(x), \quad \mathfrak{m}_{\nu_{i}, \alpha_{i}},
$$

where $\lambda, \mu, \nu_{i}$ stand for partitions. Therefore, we obtain integral classes:

$$
\frac{1}{\mathfrak{z} \lambda} \mathfrak{a}_{-\lambda}(1) \mathfrak{a}_{-\mu}(x) \mathfrak{m}_{\nu^{1}, \alpha_{1}} \cdots \mathfrak{m}_{\nu^{k}, \alpha_{k}}|0\rangle
$$

Recall from the conditions in Theorem 1.1 that

$$
H^{1}(X)=H^{3}(X)=0 .
$$

By $(2.3)$, the number of classes in $(1.1)$ is equal to the rank of $H^{*}\left(X^{[n]} ; \mathbb{Z}\right) /$ Tor. Now consider the intersection number of two classes from (1.1):

$$
\begin{aligned}
& \frac{1}{\mathfrak{z} \lambda} \mathfrak{a}_{-\lambda}(1) \mathfrak{a}_{-\mu}(x) \mathfrak{m}_{\nu^{1}, \alpha_{1}} \cdots \mathfrak{m}_{\nu^{k}, \alpha_{k}}|0\rangle, \\
& \frac{1}{\mathfrak{z} \tilde{\lambda}} \mathfrak{a}_{-\tilde{\lambda}}(1) \mathfrak{a}_{-\tilde{\mu}}(x) \mathfrak{m}_{\tilde{\nu}^{1}, \alpha_{1}} \cdots \mathfrak{m}_{\tilde{\nu}^{k}, \alpha_{k}}|0\rangle .
\end{aligned}
$$

By the Heisenberg algebra commutation relation (2.2), the intersection number is

$\left.(3.20) \pm \delta_{\lambda, \tilde{\mu}} \cdot \delta_{\tilde{\lambda}, \mu} \cdot\left\langle\mathfrak{m}_{\nu^{1}, \alpha_{1}} \cdots \mathfrak{m}_{\nu^{k}, \alpha_{k}} \mid 0\right\rangle, \mathfrak{m}_{\tilde{\nu}^{1}, \alpha_{1}} \cdots \mathfrak{m}_{\tilde{\nu}^{k}, \alpha_{k}}|0\rangle\right\rangle$.

Recall from the Modularity Theorem 4.6 in [15] that for every fixed $\tilde{n} \geq 0$, the intersection matrix formed by the pairings of the classes

$$
\mathfrak{m}_{\nu^{1}, \alpha_{1}} \cdots \mathfrak{m}_{\nu^{k}, \alpha_{k}}|0\rangle, \quad \sum_{i=1}^{k}\left|\nu^{i}\right|=\tilde{n}
$$


has determinant \pm 1 . Hence we conclude from (3.20) that the intersection matrix formed by the pairings of the integral classes in (1.1) is unimodular. Therefore, the classes in (1.1) form an integral basis of $H^{*}\left(X^{[n]} ; \mathbb{Z}\right) /$ Tor.

\section{Generators of the integral cohomology ring}

In this section, we shall assume that the odd Betti numbers of the surface $X$ are equal to zero, and study generators for the integral cohomology ring of $X^{[n]}$.

Let $\alpha_{1}, \ldots, \alpha_{k}$ be an integral basis for $H^{2}(X ; \mathbb{Z}) /$ Tor. By $(1.1)$, we have the following integral cohomology classes in $H^{*}\left(X^{[n]}\right)$ :

$$
\begin{aligned}
& \mathbf{1}_{-(n-j)} \mathfrak{m}_{\left(1^{j}\right), \alpha_{s}}|0\rangle, \\
& \mathbf{1}_{-(n-j)} \mathfrak{a}_{-j}(x)|0\rangle,
\end{aligned}
$$

where $1 \leq j \leq n$ and $1 \leq s \leq k$. Recall the tautological rank- $n$ vector bundle $\mathcal{O}_{X}^{[n]}$ defined by $(3.10)$, and note from $(3.11)$ that $c_{n}\left(\mathcal{O}_{X}^{[n]}\right)=0$.

Definition 4.1. For $n \geq 1$, we define $\mathcal{R}_{n}$ to be the subring of the integral cohomology ring $H^{*}\left(X^{[n]} ; \mathbb{Z}\right) /$ Tor generated over $\mathbb{Z}$ by all the integral classes:

$$
c_{i}\left(\mathcal{O}_{X}^{[n]}\right), \quad \mathbf{1}_{-(n-j)} \mathfrak{m}_{\left(1^{j}\right), \alpha_{s}}|0\rangle, \quad \mathbf{1}_{-(n-j)} \mathfrak{a}_{-j}(x)|0\rangle
$$

where $1 \leq i \leq n-1,1 \leq j \leq n$, and $1 \leq s \leq k$.

Remark 4.2. Fix a point $x \in X$ and a smooth irreducible curve $C \subset X$. Then the integral class $\mathbf{1}_{-(n-j)} \mathfrak{a}_{-j}(x)|0\rangle$ is represented by the subvariety

$$
\left\{\xi \in X^{[n]} \mid m(\xi, x) \geq j\right\}
$$

where $m(\xi, x)$ denotes the multiplicity of $\xi$ at the point $x$. Similarly, the integral class $\mathbf{1}_{-(n-j)} \mathfrak{m}_{\left(1^{j}\right), C}|0\rangle$ is represented by the closure of the subvariety in $X^{[n]}$ :

$$
\left\{\sum_{i=1}^{n-j} x_{i}+\sum_{i=1}^{j} x_{i}^{\prime} \mid x_{i} \notin C, x_{i}^{\prime} \in C, \text { and } x_{1}, \ldots, x_{n-j}, x_{1}^{\prime}, \ldots, x_{j}^{\prime} \text { are distinct }\right\} .
$$


Our goal in this section is to show that

$$
\mathcal{R}_{n}=H^{*}\left(X^{[n]} ; \mathbb{Z}\right) / \text { Tor }
$$

i.e., we shall prove that the ring $H^{*}\left(X^{[n]} ; \mathbb{Z}\right) /$ Tor is generated by the integral classes in (4.1). We begin with two lemmas which enable us to use certain induction.

Lemma 4.3. If $\lambda$ is a partition with $|\lambda| \leq n$, then

$$
\mathbf{1}_{-(n-|\lambda|)} \mathfrak{m}_{\lambda, \alpha_{i}}|0\rangle \equiv A\left(\bmod \mathcal{R}_{n}\right)
$$

where $A$ is an integral linear combination of classes of the form

$$
\frac{1}{\mathfrak{z} \tilde{\lambda}} \mathfrak{a}_{-\tilde{\lambda}}(1) \mathfrak{a}_{-\mu}(x) \mathfrak{m}_{\nu^{1}, \alpha_{1}} \cdots \mathfrak{m}_{\nu^{k}, \alpha_{k}}|0\rangle
$$

such that the partitions $\tilde{\lambda}=\left(1^{\tilde{m}_{1}} 2^{\tilde{m}_{2}} \ldots\right)$ satisfy $\tilde{m}_{1}>(n-|\lambda|)$, and

$$
|\tilde{\lambda}|+|\mu|+\left|\nu^{1}\right|+\cdots+\left|\nu^{k}\right|=n
$$

Proof. Combining formulas (3.6) and (3.18), we obtain

$$
\mathfrak{m}_{\lambda, \alpha_{i}}|0\rangle=\left[L^{\lambda} \alpha_{i}\right]=P\left(\mathfrak{m}_{\left(1^{j}\right), \alpha_{i}} \mid j \geq 1\right)|0\rangle
$$

where $P$ is an integral polynomial. It follows that

$$
\mathbf{1}_{-(n-|\lambda|)} \mathfrak{m}_{\lambda, \alpha_{i}}|0\rangle=\mathbf{1}_{-(n-|\lambda|)} P\left(\mathfrak{m}_{\left(1^{j}\right), \alpha_{i}} \mid j \geq 1\right)|0\rangle
$$

Therefore, it remains to prove that if $j_{1}+\cdots+j_{s}=|\lambda|$, then

$$
\mathbf{1}_{-(n-|\lambda|)} \mathfrak{m}_{\left(1^{j_{1}}\right), \alpha_{i}} \cdots \mathfrak{m}_{\left(1^{j_{s}}\right), \alpha_{i}}|0\rangle \equiv A_{1}\left(\bmod \mathcal{R}_{n}\right)
$$

where $A_{1}$ is an integral linear combination of classes of the form (4.3).

By definition, the classes $\mathbf{1}_{-\left(n-j_{u}\right)} \mathfrak{m}_{\left(1^{j_{u}}\right), \alpha_{i}}|0\rangle, 1 \leq u \leq s$, are contained in $\mathcal{R}_{n}$. By $(3.9), \mathbf{1}_{-\left(n-j_{u}\right)} \mathfrak{m}_{\left(1^{j_{u}}\right), \alpha_{i}}|0\rangle$ is a linear combination of classes of the 
form

$$
\mathbf{1}_{-\left(n-j_{u}\right)} \mathfrak{a}_{-\gamma}\left(\alpha_{i}\right)|0\rangle, \quad|\gamma|=j_{u} .
$$

By Theorem 2.1, the cup product of the classes $\mathbf{1}_{-\left(n-j_{u}\right)} \mathfrak{m}_{\left(1^{j_{u}}\right), \alpha_{i}}|0\rangle, 1 \leq$ $u \leq s$, is

$$
\mathbf{1}_{-(n-|\lambda|)} \mathfrak{m}_{\left(1^{j_{1}}\right), \alpha_{i}} \cdots \mathfrak{m}_{\left(1^{j_{s}}\right), \alpha_{i}}|0\rangle+\mathbf{1}_{-(n-|\lambda|+1)} A_{2}
$$

for some cohomology class $A_{2} \in H^{*}\left(X^{[|\lambda|-1]}\right)$. Hence, we obtain

$$
\mathbf{1}_{-(n-|\lambda|)} \mathfrak{m}_{\left(1^{j_{1}}\right), \alpha_{i}} \cdots \mathfrak{m}_{\left(1^{j_{s}}\right), \alpha_{i}}|0\rangle \equiv-\mathbf{1}_{-(n-|\lambda|+1)} A_{2}\left(\bmod \mathcal{R}_{n}\right) .
$$

Since $\mathbf{1}_{-(n-|\lambda|)} \mathfrak{m}_{\left(1^{j_{1}}\right), \alpha_{i}} \cdots \mathfrak{m}_{\left(1^{j s}\right), \alpha_{i}}|0\rangle$ is an integral class, we conclude from Theorem 1.1 that the class $A_{1}:=-\mathbf{1}_{-(n-|\lambda|+1)} A_{2}$ is an integral linear combination of classes of the form (4.3). This completes the proof of (4.4).

Lemma 4.4. Let $\lambda=\left(1^{m_{1}} 2^{m_{2}} \cdots\right)$ be a partition of $n$. Then,

$$
\frac{1}{\mathfrak{z} \lambda} \mathfrak{a}_{-\lambda}(1)|0\rangle \equiv A\left(\bmod \mathcal{R}_{n}\right)
$$

where $A$ is an integral linear combination of classes of the form

$$
\frac{1}{\mathfrak{z}_{\tilde{\lambda}}} \mathfrak{a}_{-\tilde{\lambda}}(1) \mathfrak{a}_{-\mu}(x) \mathfrak{m}_{\nu^{1}, \alpha_{1}} \cdots \mathfrak{m}_{\nu^{k}, \alpha_{k}}|0\rangle
$$

such that $\mu \neq \emptyset$ or $\nu^{i} \neq \emptyset$ for some $i, \tilde{\lambda}=\left(1^{\tilde{m}_{1}} 2^{\tilde{m}_{2}} \ldots\right)$ with $\tilde{m}_{1}>m_{1}$, and

$$
|\tilde{\lambda}|+|\mu|+\left|\nu^{1}\right|+\cdots+\left|\nu^{k}\right|=n .
$$

Proof. For simplicity, let $G_{i}=G_{i}(1, n)$ and $c_{i}=c_{i}\left(\mathcal{O}_{X}^{[n]}\right)$. By Corollary 4.8 in [17], the cohomology class $G_{r-1}$ is equal to

$$
\frac{(-1)^{r-1}}{r !} \cdot \mathbf{1}_{-(n-r)} \mathfrak{a}_{-r}(1)|0\rangle+\mathbf{1}_{-(n-r+1)} A_{1}
$$

for some class $A_{1} \in H^{*}\left(X^{[r-1]}\right)$. By Theorem 2.1, the cup product $\prod_{r \geq 2} G_{r-1}^{m_{r}}$ equals

$$
\prod_{r \geq 2}\left(\frac{(-1)^{r-1}}{r !}\right)^{m_{r}} \cdot \frac{1}{m_{1} !} \cdot \mathfrak{a}_{-\lambda}(1)|0\rangle+\mathbf{1}_{-\left(m_{1}+1\right)} A_{2}
$$

for some class $A_{2}$. In view of the basis (1.1), we can write $\mathbf{1}_{-\left(m_{1}+1\right)} A_{2}$ as

$$
\sum_{\lambda^{\prime}} d_{\lambda^{\prime}} \mathfrak{a}_{-\lambda^{\prime}}(1)|0\rangle+A_{3}
$$


where $A_{3}$ is a linear combination of classes of the form (4.6), $d_{\lambda^{\prime}} \in \mathbb{Q}$, and the partitions $\lambda^{\prime}:=\left(1^{m_{1}^{\prime}} 2^{m_{2}^{\prime}} \ldots\right)$ satisfy $m_{1}^{\prime}>m_{1}$. So

$$
\prod_{r \geq 2} G_{r-1}^{m_{r}}=\frac{1}{m_{1} !} \cdot \prod_{r \geq 2}\left(\frac{(-1)^{r-1}}{r !}\right)^{m_{r}} \cdot \mathfrak{a}_{-\lambda}(1)|0\rangle+\sum_{\lambda^{\prime}} d_{\lambda^{\prime}} \mathfrak{a}_{-\lambda^{\prime}}(1)|0\rangle+A_{3} .
$$

Solving $\mathfrak{a}_{-\lambda}(1)|0\rangle$ and using induction on $m_{1}$, we conclude that

$$
\frac{1}{\mathfrak{z} \lambda} \mathfrak{a}_{-\lambda}(1)|0\rangle=P\left(G_{1}, G_{2}, \ldots\right)+A
$$

where $A$ is a linear combination of classes of the form (4.6), and $P\left(G_{1}\right.$, $\left.G_{2}, \ldots\right)$ denotes a polynomial in the classes $G_{1}, G_{2}, \ldots$ Note from (2.4) that $G_{i}$ is the $i$ th Chern character of the tautological bundle $\left(\mathcal{O}_{X}\right)^{[n]}$. Replacing the Chern characters $G_{i}$ of $\left(\mathcal{O}_{X}\right)^{[n]}$ by its Chern classes $c_{i}$, we have

$$
\frac{1}{\mathfrak{z} \lambda} \mathfrak{a}_{-\lambda}(1)|0\rangle=\widetilde{P}\left(c_{1}, c_{2}, \ldots\right)+A
$$

where $\widetilde{P}\left(c_{1}, c_{2}, \ldots\right)$ denotes a polynomial in the classes $c_{1}, c_{2}, \ldots$, and has coefficients independent of the smooth surface $X$.

Now, from the proof of the Proposition 6.1 in [8], we see that in the cohomology ring $H^{*}\left(\left(\mathbb{C}^{2}\right)^{[n]}\right)$ of the Hilbert scheme $\left(\mathbb{C}^{2}\right)^{[n]}$, we also have

$$
\frac{1}{\mathfrak{z} \lambda} \mathfrak{a}_{-\lambda}(1)|0\rangle=\widetilde{P}\left(\tilde{c}_{1}, \tilde{c}_{2}, \ldots\right)
$$

where $1 \in H^{0}\left(\mathbb{C}^{2}\right)$ is the identity element, and $\tilde{c}_{i}$ denotes the $i$ th Chern class of the tautological bundle $\left(\mathcal{O}_{\mathbb{C}^{2}}\right)^{[n]}$ on $\left(\mathbb{C}^{2}\right)^{[n]}$. Moreover, all the coefficients in the polynomial $\widetilde{P}\left(\tilde{c}_{1}, \tilde{c}_{2}, \ldots\right)$ are integers. Thus by $(4.7)$, we obtain

$$
\frac{1}{\mathfrak{z} \lambda} \mathfrak{a}_{-\lambda}(1)|0\rangle \equiv A\left(\bmod \mathcal{R}_{n}\right)
$$

Finally, note that $1 / \mathfrak{z}_{\lambda} \mathfrak{a}_{-\lambda}(1)|0\rangle$ and all the classes in $\mathcal{R}_{n}$ are integral. Since $A$ is a linear combination of the classes (4.6), we conclude from Theorem 1.1 that all the coefficients in this linear combination are integers.

Now we can present a proof of Theorem 1.2. 
Proof of Theorem 1.2. By Theorem 1.1, it suffices to show that all the basis classes (1.1) are contained in $\mathcal{R}_{n}$. These basis classes can be rewritten as

$$
\mathbf{1}_{-\left(n-n_{0}\right)} \frac{1}{\mathfrak{z} \rho} \mathfrak{a}_{-\rho}(1) \mathfrak{a}_{-\mu_{1}}(x) \cdots \mathfrak{a}_{-\mu_{\ell}}(x) \mathfrak{m}_{\nu^{1}, \alpha_{1}} \cdots \mathfrak{m}_{\nu^{k}, \alpha_{k}}|0\rangle,
$$

where $0 \leq n_{0} \leq n$, the partition $\rho$ is of the form $\left(2^{m_{2}} 3^{m_{3}} \cdots\right)$ and

$$
|\rho|+\left(\mu_{1}+\cdots+\mu_{\ell}\right)+\sum_{i=1}^{k}\left|\nu^{i}\right|=n_{0} .
$$

In the following, we shall use induction on $n_{0}$ to prove that

$$
\text { (4.8) } \quad A:=\mathbf{1}_{-\left(n-n_{0}\right)} \frac{1}{\mathfrak{z} \rho} \mathfrak{a}_{-\rho}(1) \mathfrak{a}_{-\mu_{1}}(x) \cdots \mathfrak{a}_{-\mu_{\ell}}(x) \mathfrak{m}_{\nu^{1}, \alpha_{1}} \cdots \mathfrak{m}_{\nu^{k}, \alpha_{k}}|0\rangle \in \mathcal{R}_{n}
$$

Note that (4.8) is trivially true when $n_{0}=0$. Now fix $n_{0}>0$, and assume that

$$
\mathbf{1}_{-\left(n-\tilde{n}_{0}\right)} \frac{1}{\mathfrak{z} \tilde{\rho}} \mathfrak{a}_{-\tilde{\rho}}(1) \mathfrak{a}_{-\tilde{\mu}_{1}}(x) \cdots \mathfrak{a}_{-\tilde{\mu}_{\tilde{\ell}}}(x) \mathfrak{m}_{\tilde{\nu}^{1}, \alpha_{1}} \cdots \mathfrak{m}_{\tilde{\nu}^{k}, \alpha_{k}}|0\rangle \in \mathcal{R}_{n}
$$

holds for all $\tilde{n}_{0}<n_{0}$. We want to verify that (4.8) is also true.

We start with the three special cases when $\mu_{1}=n_{0}($ so $\ell=1)$, or $|\rho|=$ $n_{0}$, or $\left|\nu^{i}\right|=n_{0}$ for some $i$. First of all, by the definition of $\mathcal{R}_{n}$, we have

$$
\mathbf{1}_{-\left(n-n_{0}\right)} \mathfrak{a}_{-n_{0}}(x)|0\rangle \in \mathcal{R}_{n}
$$

Next, we see from Lemma 4.4 and the induction hypothesis that

$$
\mathbf{1}_{-\left(n-n_{0}\right)} \frac{1}{\mathfrak{z}_{\rho}} \mathfrak{a}_{-\rho}(1)|0\rangle \in \mathcal{R}_{n}
$$

for every partition $\rho=\left(2^{m_{2}} 3^{m_{3}} \ldots\right)$ with $|\rho|=n_{0}$. Finally, for every partition $\nu^{i}$ with $\left|\nu^{i}\right|=n_{0}$, we conclude from Lemma 4.3 and the induction hypothesis that

$$
\mathbf{1}_{-\left(n-n_{0}\right)} \mathfrak{m}_{\nu^{i}, \alpha_{i}}|0\rangle \in \mathcal{R}_{n}
$$

For general $\rho, \mu_{1}, \ldots, \mu_{\ell}, \nu^{1}, \ldots, \nu^{k}$ from (4.8), we consider the classes:

$$
\begin{gathered}
\mathbf{1}_{-(n-|\rho|)} \frac{1}{\mathfrak{z} \rho} \mathfrak{a}_{-\rho}(1)|0\rangle, \\
\mathbf{1}_{-\left(n-\mu_{i}\right)} \mathfrak{a}_{-\mu_{i}}(x)|0\rangle \quad(1 \leq i \leq \ell), \\
\mathbf{1}_{-\left(n-\left|\nu^{i}\right|\right)} \mathfrak{m}_{\nu^{i}, \alpha_{i}}|0\rangle \quad(1 \leq i \leq k),
\end{gathered}
$$


which are contained in $\mathcal{R}_{n}$ by the preceding paragraph. By Theorem 2.1, their cup product is $A+\mathbf{1}_{-\left(n-n_{0}+1\right)} A_{1}$ for some class $A_{1} \in H^{*}\left(X^{\left[n_{0}-1\right]}\right)$. Therefore,

$$
A \equiv-\mathbf{1}_{-\left(n-n_{0}+1\right)} A_{1}\left(\bmod \mathcal{R}_{n}\right) .
$$

Note that the integral class $-\mathbf{1}_{-\left(n-n_{0}+1\right)} A_{1}$ is an integral linear combination of classes of the form (4.9). Hence by induction, we have $A \in \mathcal{R}_{n}$.

\section{Acknowledgments}

We thank F. Bogomolov, E. Markman and Weiqiang Wang for valuable discussion and suggestion. We also thank the referee for valuable comments. The second author thanks Hong Kong University of Science and Technology for its hospitality during his visit in March 2004, while the first author thanks the University of Missouri for its hospitality during his visit in February 2006. The first author was partially supported by the grant HKUST6114/02P and CERG601905. The second author was partially supported by an NSF grant.

\section{References}

[1] L. Göttsche, The Betti numbers of the Hilbert scheme of points on a smooth projective surface, Math. Ann. 286 (1990), 193-207.

[2] I. Grojnowski, Instantons and affine algebras I: the Hilbert scheme and vertex operators, Math. Res. Lett. 3 (1996), 275-291.

[3] H. Nakajima, Heisenberg algebra and Hilbert schemes of points on projective surfaces, Ann. Math. 145 (1997), 379-388.

[4] G. Ellingsrud and S. Strømme, Towards the Chow ring of the Hilbert scheme of $\mathbb{P}^{2}$, J. Reine Angew. Math. 441 (1993), 33-44.

[5] A. Beauville, Sur la cohomologie de certains espaces de modules de fibrés vectoriels, Geometry and Analysis, Bombay, 1992, Tata Inst. Fund. Res., Bombay, 1995, 37-40.

[6] W.-P. Li, Z. Qin and W. Wang, Vertex algebras and the cohomology ring structure of Hilbert schemes of points on surfaces, Math. Ann. 324 (2002), 105-133.

[7] M. Lehn, Chern classes of tautological sheaves on Hilbert schemes of points on surfaces, Invent. Math. 136 (1999), 157-207. 
[8] M. Lehn and C. Sorger, Symmetric groups and the cup product on the cohomology of Hilbert schemes, Duke Math. J. 110 (2001), 345-357.

[9] - The cup product of the Hilbert scheme for K3 surfaces, Invent. Math. 152 (2003), 305-329.

[10] E. Vasserot, Sur l'anneau de cohomologie du schéma de Hilbert de $\mathbb{C}^{2}$, C. R. Acad. Sci. Paris, Sér. I Math. 332 (2001), 7-12.

[11] L. Göttsche, The cohomology ring of the Hilbert scheme of points on a surface, Talk at the International Conference in Algebraic Geometry, Shanghai, August 2002.

[12] E. Markman, Generators of the cohomology ring of moduli spaces of sheaves on symplectic surfaces, J. Reine Angew. Math. 544 (2002), 61-82.

[13] - Integral generators for the cohomology ring of moduli spaces of sheaves over Poisson surfaces, Adv. Math. 208 (2007), 622-646.

[14] - Integral constraints on the monodromy group of the hyperkahler resolution of a symmetric product of a K3 surface, Preprint. ArXiv:math/0601304.

[15] Z. Qin and W. Wang, Integral operators and integral cohomology classes of Hilbert schemes, Math. Ann. 331 (2005), 669-692.

[16] H. Nakajima, Lectures on Hilbert schemes of points on surfaces, Univ. Lect. Ser., 18, Amer. Math. Soc., R.I., 1999.

[17] W.-P. Li, Z. Qin and W. Wang, Hilbert schemes and $\mathcal{W}$ algebras, Intern. Math. Res. Notices. 27 (2002), 1427-1456.

[18] - Stability of the cohomology rings of Hilbert schemes of points on surfaces, J. Reine Angew. Math. 554 (2003), 217-234.

[19] I. G. Macdonald, Symmetric functions and Hall polynomials, 2nd ed., Clarendon Press, Oxford, 1995.

[20] Z. Qin and W. Wang, Hilbert schemes and symmetric products: a dictionary, in Proceedings for "Mathematical Aspects of Orbifold String Theory", eds. A. Adem et al, (eds) Madison, Wisconsin, May 2001; Contemp. Math. 310 (2002), 233-257.

[21] M.F. Atiyah and F. Hirzebruch, Vector bundles and homogeneous spaces, Proc. Sympos. Pure Math, 3, American Mathematical Society, Providence, RI, 1961, 7-38. 
[22] D. Freed and K. Uhlenbeck, Instantons and four-manifolds, M.S.R.I. Publications, 1, Springer-Verlag, New York, 1991.

Department of Mathematics HKUST

Clear Water Bay, Kowloon

HONG KONG

E-mail address: mawpli@ust.hk

Department of Mathematics

UNIVERSITY OF MISSOURI

Columbia, MO 65211

USA

E-mail address: zq@math.missouri.edu

ReCEIVED July 1, 2006 